\title{
Guest Editor's Introduction
}

\author{
Yong Qin \\ Beijing Jiaotong University \\ Beijing, P. R. China \\ yqin@bjtu.edu.cn \\ $\operatorname{Min} \mathrm{An}^{*}$ \\ University of Salford \\ Manchester, UK \\ M.An@salford.ac.uk \\ Limin Jia \\ Beijing Jiaotong University \\ Beijing, P. R. China \\ lmjia@bjtu.edu.cn \\ Published
}

\section{Description}

Railways are by far one of the safest means of ground transportation, especially for their passengers and employees. However, there are serious issues involved in b maintaining this position in reality and sustaining the public perception of excell railway safety. The railway now finds itself in a situation where actual and perceived safeties are real issues, to be dealt with in a new public culture of rapid change, shortapprove edit. term pressures, and instant communications.

Railway safety is a very complicated subject, which is determined by numerous aspects including the application of advanced technologies. Technologies applied in railway transportation development require accurate modeling and simulation of rail network, train and passenger flows which involve information acquisition, data fusion, state identification, knowledge inference, system optimization and control. This presents a higher combination and coordination between physical and

${ }^{*}$ Corresponding author. 


AQ: Please
check the
LRH.

\section{Y. Qin M.An \& L. Ji}

computational elements in rail and vehicle design, construction, operation, maintenance and management. This special issue focuses on advanced mathematic $\bigcirc$ AQ: Please modeling and optimal control in the railway domain and its related transport ne 2 works. The 4th International Conference on Electrical Engineering and Information approve edits. Technologies for Rail Transportation (EITRT2019) in Qingdao, Shandong Province, China, 25-27 October 2019 brought together worldwide practitioners, leading researchers and postgraduates showcasing state-of-the-art research, innovation and industry practice at all levels. The aim is to identify new technologies and methods for improving the effectiveness and efficiency of railway design, construction, operation, maintenance and management.

This special issue is to select a compendium of high-quality research papers from EITRT2019 as well as review articles addressing recent theoretical and practical advances on railways including engineering, information technologies and sciences, software engineering and knowledge engineering for rail transportation. We expect contributions that represent state-of-the-art approaches and breakthroughs in mathematical modeling, simulation, and particularly optimization to tackle railway engineering, operation, maintenance and management challenges. Submitted articles should be original and not yet published contributions or contributions that are not currently under review by any other journals.

Papers are invited on research in the following four areas related to rail transportation, including but not limited to the following topics.

AREA 1 - Novel Traction Drive Technologies of Rail Transportation. Potential topics include:

- New power electronic devices and applications,

- Power quality management,

- High quality current-carrying technology,

- Advance micro-control systems,

- Traction motor control,

- High frequency of power electronics,

- Efficiency-increasing control for traction systems,

- Traction safety and control technologies under complex conditions,

- Electric braking security,

- Power electronic system RAMS,

- Error detection, diagnosis and remaining life prediction,

- Hybrid traction technologies,

- Energy saving and emission reduction,

- Application of Lithium battery and super capacitor, etc.,

- New traction power supply systems,

- New traction drive systems. 
AREA 2 - Rail Transportation System Safety and Maintenance Technology. Potential topics include:

- Rail transportation safety and risk analysis,

- Train operation safety monitoring, pre-warning and maintenance,

- Infrastructure security test and maintenance,

- Natural environment and perimeter intrusion security technology,

- Passenger flow and human factor safety technology,

- PHM technology in rail transportation,

- Safety modeling and simulation,

- Fault detection, diagnosis and forecast,

- Rail transportation system RAMS,

- Emergency preparedness and management.

$A R E A 3$ - Rail Transportation Information Technology. Potential topics include:

- Intelligent railway system and technology,

- Big data analysis technology,

- CPS information fusion technology,

- Cloud/edge computing technology,

- Internet of things/sensor network technology,

- Artificial intelligence technology,

- Video/voice processing technology,

- New technology of train network,

- Next generation communication technology,

- Advanced/unmanned train operation control technology,

- Network information security technology.

AREA 4 - Rail Transportation Operation and Management Technology. Potential topics include:

- System modeling and analysis,

- Analysis and forecasting technology of passenger and freight demand,

- Optimization of train operation planning,

- Train dispatch, command and emergency response,

- Analysis, forecast and control technology of passenger flow,

- Railway logistics monitoring command and automation technology,

- Station and operation automation technology,

- Station operation management and automation equipment.

\section{Guest Introduction}

Rail transportation is now facing significant challenges due to system complexity, applications of advanced and artificial intelligent technologies in design, 
construction, operation, maintenance and management which involve information acquisition, data fusion, state identification, knowledge inference, system optimization and control. Therefore, an interdisciplinary approach is needed to address these challenges, and electrical engineering and information technologies are emerging as leading contributors to the future of rail transportation. The 4th Internati Conference on Electrical Engineering and Information Technologies for Rail Transportation (EITRT2019) in Qingdao, Shandong Province, China, 25-27 October 2019 brought together worldwide practitioners, leading researchers and postgraduates showcasing state-of-the-art research, innovation and industry practice at all levels and in all processes in the electrical engineering, information technologies and sciences, software engineering and knowledge engineering for rail transportation. In this context, four research papers included in this special issue of IJSEKE are selected from 172 accepted papers at EITRT2019 on the basis of a peer review process with an aim at providing a collection of innovative and high-quality research contributions focusing on the application of knowledge engineering techniques to software simulation engineering in railways to demonstrate the state-of-the-art of remote sensing and intelligent monitoring for rail transportation. All of authors of these papers have been required to extend, revise and update their papers to meet scopes and themes of the IJSEKE, and journal publication quality through a norm $\mathbb{Q}$ journal paper review process. Special Issues of IJSEKE accepted for publication are summarized below.

The paper entitled "An Integration of Train Timetabling, Platforming and Routing Based Cooperative Adjustment Methodology for Dealing with Train Delay" by Yinggui Zhang, Zengru Chen, Min An and Aliyu Mani Umar presents a methodology by applying intelligent railway monitor and control system to improve train operation management while increasing railway safety in which train timetabling, platforming and routing models are combined by studying the real-time adjustment and optimization of high-speed railway in the case of the train delay in order to produce a cooperative adjustment algorithm so that the train operation adjustment plan can be obtained. MATLAB computer programs have been developed based on the proposed methodology and adjustment criteria have been established from knowledge data bases in order to search for optimized solutions effectively and efficiently. A case study is used to demonstrate the application of the proposed methodology.

Accurate prediction of train delay recovery is critical for railway incident management and will provide the information of journey time for passengers. The paper by Bowen Gao, Dongxiu Ou, Decun Dong and Yusen Wu entitled "A Data-driven Two-stage Prediction Model for Train Primary-delay Recovery Time" proposes a model to deal with train delay recovery. A detailed presentation of the proposed model design and development is presented in the article. A validation test case is used for testing the model, and the results produced from the proposed model are then compared with the results from logistic regression (LR), artificial neutral

AQ: Please
check for
repetition.


network (ANN), support vector machine (SVM) and gradient booting tree (GBT) methods.

A deep-learning-based method with a top-down-top feature pyramid structure for dealing with railway infrastructure high level semantic and low level detail information acquired by unmanned aerial vehicle is presented in the paper "An Improved Faster R-CNN for UAV-based Catenary Support Device Inspection" by Jiahao Liu, Zhipeng Wang, Yunpeng Wu, Yong Qin, Xianbin Cao and Yonghui Huang. This paper introduces the recent applications of railway infrastructure intelligent monitoring technology via UAV in China high speed rails. The proposed method can detect catenary support device defect using UAV images based on the improved Faster R-CNN model. More details of experiment equipment setting, dataset development, deep neural network training process and results are described in the paper.

The paper "The Turnout Abnormality Diagnosis with Semi-supervised Learning Method" by Zengshu Shi, Yiman Du, Tao Du and Guochao Shan explores turnout action curves, and static and dynamic properties that have been collected from railway centralized monitoring system, and develops a methodology based on semisupervised learning algorithm for diagnosis data and information analysis. Simulations with a large number of training and testing sets show that by using the proposed method, the turnout electrical characteristics can be accurately diagnosed, which can enhance railway safety and reduce rail transportation accidents.

We hope the readers of IJSEKE will find useful information and benefits from these papers.

\section{Conflicts of Interest}

The quest editors declare that there are no conflicts of interest regarding this special issue.

\section{Acknowledgments}

We would like to express our appreciation to all the authors, reviewers, and editorial team for great support to make this special issue possible. Special thanks to Professor Shi-Kuo Chang, Editor-in-Chief of IJSEKE for his support and invaluable suggestions and advice to ensure the successful publication of this special issue. 